\title{
Mango seed fibers surface chemical modification applied as mechanical reinforcement in cementitious composites
}

\begin{abstract}
As well known, the fibers can be used as mechanical reinforcement fillers in the composites. In the specific case of vegetable fibers in cementitious matrices, there are some concerns about low adhesion on the matrix, high water absorption by the fiber and low durability in alkaline media, which can be improved by some chemical treatments on the fiber surface. This present work aim to evaluate a single coating of a magnetite layer and a double coating of polyaniline layer and magnetite layer on the mango seed fiber surface. These coating aimed to get better the fiber/matrix adhesion as well as to promote a chemical protection of the fiber. The natural and coated mango seed fibers were characterized by FTIR and TGA, to evaluate the coating process result. The morphology of the composites presents no change using natural or coated fibers. The mechanical properties of the composites containing the fibers, natural and coated, were determined by compression tests. The fibers presence, independent on the surface treatment, got better the all composites mechanical properties. On the other hand, the composite with $0.5 \%$ of double coated fibers had similar young's modulus than natural fiber, associated the chemical protection on the fiber.
\end{abstract}

Keywords: mango seed fiber, chemical modification, magnetite, polyaniline, mechanical reinforcement
Volume I Issue 5 - 2017

\author{
Moura BR,' Oliveira GE, ${ }^{2}$ Toledo Filho RD, ${ }^{2}$ \\ Souza FG', \\ 'Instituto de Macromoléculas, Universidade Federal do Rio de \\ Janeiro(IMA/UFRJ), Brazil \\ ${ }^{2}$ Programa de Engenharia Civil, Universidade Federal do Rio de \\ Janeiro(PEC /COPPE/UFRJ), Brazil
}

Correspondence: Oliveira GE, Programa de Engenharia Civil, Universidade Federal do Rio de Janeiro(PEC / COPPE/UFRJ), Av. Horácio de Macedo, 2030, I- I I0, Cidade Universitária, Rio de Janeiro-RJ, Brazil, Fax 21941-590,

Email geizaesperandio@gmail.com

Received: October 28, 2017 | Published: November 29, 2017
Abbreviations: Mag, magnetite; PAni, polyaniline; APS, ammonium persulfate; FTIR, fourier transformed infrared; TGA, thermal gravimetric analysis; PVA, poly (vinyl alcohol); PP, poly propylene

\section{Introduction}

Cementitious composite is one of the most widely used construction materials in the world because its abundant resources, strong adaptability and mature production process. ${ }^{1}$ Despite cementitious matrices present good compression strength, they are brittle and usually break when suffer some strain caused by tensile effort. Aiming to overpass this problem some fibers can be used as reinforcement, being the most commonly used fibers of steel, glass, poly (vinyl alcohol) (PVA), polypropylene (PP) and carbon. ${ }^{2}$ However, vegetable fibers, also named lignocellulosic fibers, has been used for this application due to their biodegradability, abundance, considering they are from renewable sources, low cost and low health risk. As disadvantage the vegetable fibers present low adhesion on the cementitious matrix, high water absorption by the fiber and low durability in alkaline media, related to the hemicelluloses and lignin degradation followed by fibers mineralization. ${ }^{3}$

The mechanical properties of a vegetable fiber-reinforced composite depend on many parameters, such as fiber strength, modulus, fiber length and orientation, in addition to the fiber-matrix interfacial bond strength. A strong fiber-matrix interface bond is critical for high mechanical properties of the composites. A good interfacial bond is required for effective stress transfer from the matrix to the fiber whereby maximum utilization of the fiber strength in the composite is achieved. Modification to the fiber also improves resistance to moisture induced degradation of the interface and the composite properties. In addition, factors like processing conditions/ techniques have significant influence on the mechanical properties of fiber reinforced composites. ${ }^{4}$ To achieve the desired properties, mainly to improve the adhesion between the fibers with the cementitious matrix, some surface treatments have been studied. ${ }^{5-8}$

In this work, mango seed fibers surface were chemically modified by the coating of a magnetite layer and a double coating of polyaniline layer and magnetite layer. The mango seed fiber was chosen because they are very abundant in Brazil and their industrial process produces from 30 to $40 \%$ of wastes, which means thousands of waste tons per year. ${ }^{9}$ The mango seed fiber was obtained from a simple washing and filtering process after the extraction of the fruit pulp. This surface modification aimed to get better the fiber/matrix adhesion as well as to promote a chemical protection of the fiber. The fibers, before and after the surface modifications, were characterized by FTIR and TGA, to make sure the efficiency of surface modification. After that, the fibers were used as filler in a cementitious matrix and their mechanical resistance was tested by compression evaluation. The fibers presence has improved the compression resistance of all composites; however the double coated fiber presented similar young's modulus than natural fiber, but associated the chemical protection on the fiber.

\section{Materials and methods}

\section{Materials}

Mango seed fibers were extracted from some fruits collected straightly from a mangoes tree. The fruit extract was removed leaving the fibers. They were washed and dried to be used. All used reactants in the modifications are P.A. and they were supplied by Vetec. The used water was in house distillated. 


\section{Chemical mofification of mango seed fibers}

The chemical modification with polyaniline was performed by immersion of the fibers in a $1 \mathrm{M} \mathrm{H}_{2} \mathrm{SO}_{4}$ solution and $5.37 \times 10^{-3} \mathrm{~mol}$ of aniline was slowly added. The solution was cooled down to $0^{\circ} \mathrm{C}$ with the help of an ice bath. After $2 \mathrm{~h}, 10 \mathrm{ml}$ of aqueous $\mathrm{H}_{2} \mathrm{SO}_{4}(1 \mathrm{M})$ and APS $(1 \mathrm{M})$ was added into the cooled mixture under continuous stirring for 24h. Afterwards, the fibers coated with Pani (Pani fibers) were washed, filtered and dried at $50^{\circ} \mathrm{C}$ up to constant weight. ${ }^{10-1}$

Aqueous solutions of $\mathrm{FeSO}_{4}(1 \mathrm{M})$ and $\mathrm{Fe}_{2} \mathrm{O}_{3}(1 \mathrm{M})$ were mixture in a beaker containing distillated water, under continuous stirring. The natural and PAni fibers were soaked in this beaker for $1 \mathrm{~h}$. After that it was added $\mathrm{KOH}(2 \mathrm{M})$ aqueous solution in the system to precipitate the magnetite nanoparticles, still under and continuous stirring for $30 \mathrm{~min}$. The obtained fibers, coated with a single layer of magnetite (named Mag fiber) and a double layer (named Pani+Mag fiber) were washed several times with water and finally dried at $70^{\circ} \mathrm{C}$ in an oven up to constant weigh.

\section{Composites preparation}

The composites were prepared using a matrix of cement paste with 0.4 of water/cement relation. Three amounts of mango seed fibers, before and after the chemical modifications, were added in the matrix: $0.5 \%, 1.0 \%$ and $2.0 \% \mathrm{v} / \mathrm{wt}$. The specimens were cured for 7 days in the fog room at room temperature. All composites were tested in five replications.

\section{Characterization of fibers}

FTIR spectra were performed using a Varian spectrometer, model 3100 FTIR Excalibur and ATR with a ZnSe crystal. The range measured was from 4000 to $600 \mathrm{~cm}^{-1}$, with $4 \mathrm{~cm}^{-1}$ of resolution and 60 accumulated scans. TGA analyses were carried out in a TA Q500 instrument for all samples, with a temperature range from 25 to $700^{\circ} \mathrm{C}$, a heating rate of $10^{\circ} \mathrm{C} / \mathrm{min}$ under a nitrogen flow of $150 \mathrm{~cm}^{3} / \mathrm{min}$ and alumina pan. The morphology of the composites were determined using a scanning electronic microscope (SEM) from Hitachi model TM3000, under vacuum with a secondary electron detector from Everhart-Thornley-ETD and voltage of $15 \mathrm{kV}$.

\section{Mechanical Properties}

The diametric compressive strength tests of the proof bodies were carried out using an electromechanical Shimadzu AG-X100kN with a load cell of $1 \mathrm{kN}$ and a displacement rate of $0.3 \mathrm{~mm} / \mathrm{min}$. It was tested cylindrical proof bodies with dimensions of $25 \times 25 \mathrm{~mm}$.

\section{Results and discussion}

\section{Characterization of fibers}

The surface chemical modification process efficiency was evaluated by FTIR and TGA. The FTIR spectra of Natural, Mag and Mag+PAni mango seed fibers are shown in the Figure 1. The mango seed fiber presents a typical lignocellulosic material FTIR spectrum. The wide characteristic band around $3355 \mathrm{~cm}^{-1}$ is attributed to the stretching of OH. The doublet at 2918 and $2848 \mathrm{~cm}^{-1}$ is assigned to $\mathrm{C}-\mathrm{H}$ stretching. The characteristic band at 1752 and $1646 \mathrm{~cm}^{-1}$ are assigned to $\mathrm{C}=\mathrm{O}$ stretching from ester and conjugated $\mathrm{C}=\mathrm{O}$ aldehyde and $\mathrm{C}=\mathrm{C}$ stretching, respectively. The aromatic skeleton stretching from the ring appears at $1428 \mathrm{~cm}^{-1}$, while $\mathrm{O}-\mathrm{H}$ scissoring deformation in the plane of the ring can be seen at $1321 \mathrm{~cm}^{-1}$. The $\mathrm{C}-\mathrm{H}$ stretching is observed at $1374 \mathrm{~cm}^{-1}$. The characteristic band at $1222 \mathrm{~cm}^{-1}$ is ascribed as $\mathrm{C}=\mathrm{O}$ aromatic stretching. The characteristic band at $1182 \mathrm{~cm}^{-1}$ is associated with $\mathrm{C}-\mathrm{O}-\mathrm{C}$ asymmetric stretching, while symmetric stretching of this same bond can be noticed at 1112 and $1172 \mathrm{~cm}^{-1}$. The characteristic band at $1165 \mathrm{~cm}^{-1}$ is $\mathrm{O}-\mathrm{C}-\mathrm{C}$ stretching together with the band at $1036 \mathrm{~cm}^{-1}$, being the band at $1032 \mathrm{~cm}^{-1}$ the conjugated $\mathrm{C}-\mathrm{O}-\mathrm{C}$ and O-C-C. The characteristic bands at 895 and $653 \mathrm{~cm}^{-1}$ are related to $\mathrm{C}-\mathrm{H}$ scissoring deformation in the aromatic ring. The single coated of magnetite, Mag fibers, increased the intensity of characteristic bands of the $\mathrm{O}-\mathrm{H}$ bonds, due to iron oxidation process. On the other hand, the double coated of polyaniline and magnetite, Pani+Mag fibers, promoted the reduction of the intensity of the lignocellulosic material and the $\mathrm{O}-\mathrm{H}$ bonds, pointing at this kind of coating is able to protect the fiber. ${ }^{12-14}$

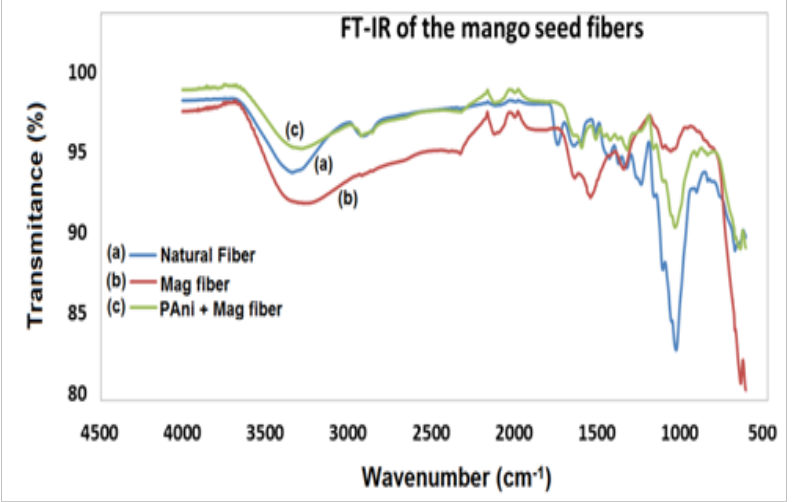

Figure I FTIR Spectra of mango seed fiber:(A) natural, (B) Mag, (C) PAni+Mag fibers.

The TGA results presented in the Figure 2 thermo gram in three steps of weight loss associated with the vaporization of water, up to $100^{\circ} \mathrm{C}$, hemicelluloses degradation, from 250 to $350^{\circ} \mathrm{C}$, and cellulose decomposition, from 325 to $400^{\circ} \mathrm{C} .{ }^{15}$ The natural was completely degraded up to $550^{\circ} \mathrm{C}$. Both single coated and double coated promoted no significant change in the degradation stages of the fibers, however, as expected, the amount of ashes has increased by 33 and $41 \%$ for Pani+Mag fibers and Mag fibers, respectively, indicating that a higher amount of magnetite coated the natural fiber than the fiber previously coated with Pani. The morphology of the composites is shown in Figure 3 by SEM images. The matrix presents a typical morphology of cementitious materials. Besides that, the morphology of composites is not change by the chemical modification of the fibers.

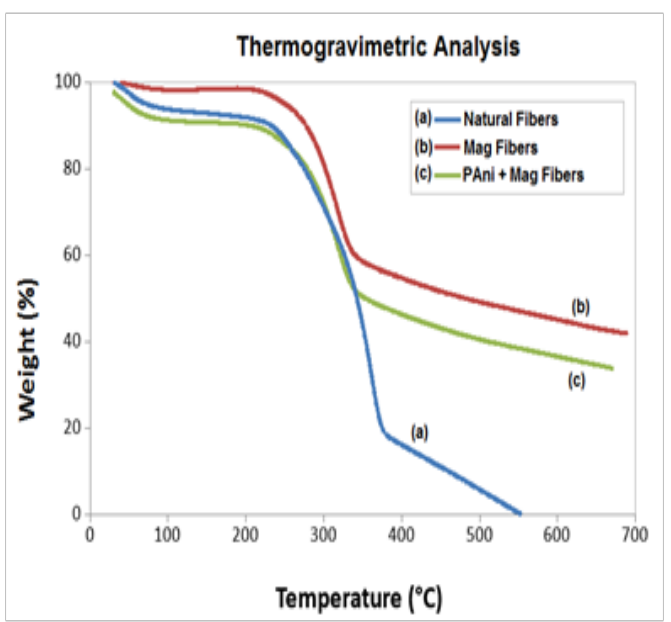

Figure 2 TGA of mango seed fiber: (A) natural, (B) Mag, (C) PAni+Mag fibers. 

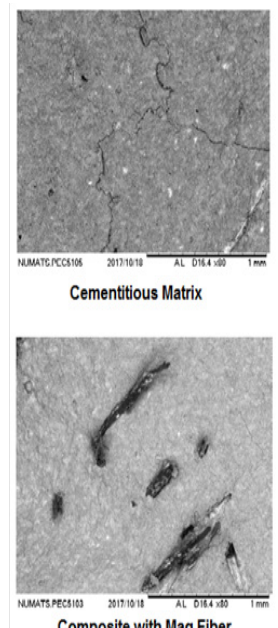

Composite with Mag Fiber
Cementitious Matrix

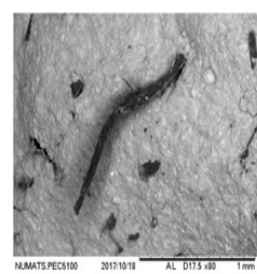

Composite with Natural Fiber

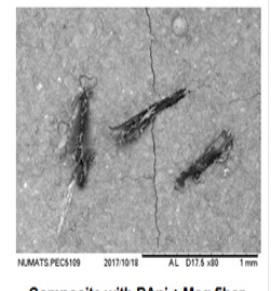

Figure 3 SEM images of the cementitious matrix, composite with natural fibers, composite with Mag fibers and composite with PAni+Mag fibers.

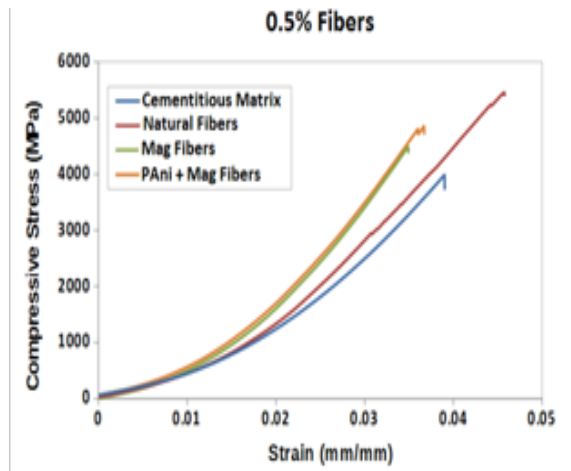

\section{Mechanical Properties}

The average stress-strain behavior curves of the composites containing $0.5 \%, 1.0 \%$ and $2.0 \%$ of natural, Mag and Pani+Mag fibers are shown in Figure 4. The average stress-strain behavior curve of the cementitious matrix is also shown as comparing. The mango seed fibers presence in cementitious composites, even without any surface modification, produced an increment in the mechanical resistance by $36 \%$, but their presence causes a reduction in the young's modulus by $8 \%$. It is an expected result considering the fibers, even being short ones, are able to transfer part of the applied stress from matrix to them. After the single coating layer of magnetite the increment in the mechanical resistance was $14 \%$, however the reduction in young's modulus was only $1 \%$. About the double coated fiber was achieved an increasing in the mechanical resistance by $22 \%$ with a no significant reduction in the young's modulus by $0.6 \%$. However it is important to highlight that this fiber presented a chemical protection on the fiber due to this kind of coating.

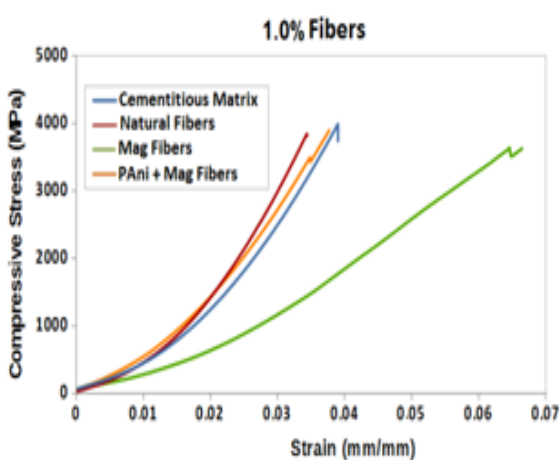

$2.0 \%$ Fibers

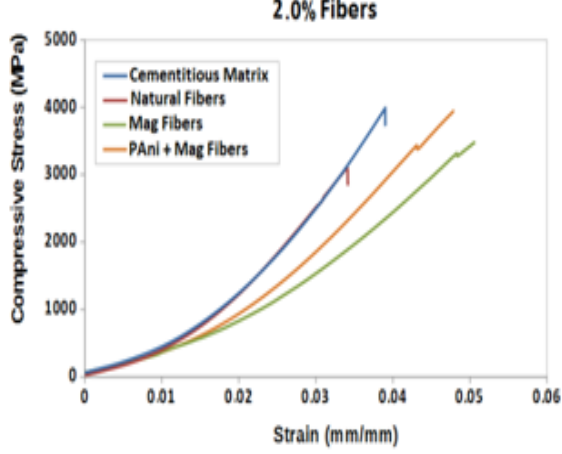

Figure 4 Stress - strain behaviour curves of cementitious matrix and composites with $0.5 \%, 1.0 \%$ and $2.0 \%$ of natural, Mag and Pani+Mag fibres.

\section{Conclusion}

Surface chemical modifications of mango seed fibers showed to be efficient, as present by the FTIR and TGA results. The main property achieved by the surface modification was the chemical protection on the fibers. It is an important property considering the aggressive environment the cementitious matrix can be for the vegetable fibers. The fiber presence caused an increment in the mechanical resistance of all composites. On the other hand, the young's modulus showed a smooth reduction when natural fiber was used and no significant change when the modified fibers were used. This result makes the double coated fiber he most appropriated filler for this kind of composite, because it kept close the mechanical properties values associated with the chemical protection of the vegetable fiber. Even the single coating has not provided good result it will be important for the next step, which is aligning the modified fibers using a magnetic field.

\section{Acknowledgements}

The authors would like to thank to Conselho Nacional de Desenvolvimento Científico e Tecnológico (CNPq), Coordenação de Aperfeiçoamento de Pessoal de Nível Superior (CAPES), Financiadora de Estudos e Projetos (FINEP) and Fundação de Amparo à Pesquisa do Estado do Rio de Janeiro (FAPERJ) for financial support and scholarships. 


\section{Conflict of interest}

The authors would like to state there is no conflict of interest.

\section{References}

1. Cui X, Han B, Zheng Q, et al. Mechanical properties and reinforcing mechanisms of cementitious composites with different types of multiwalled carbon nanotubes. Comp Part A: App Sci \& Manuf. 2017;103:131-147.

2. Santos CC, Rodrigues JPC. Compressive strength at high temperatures of a concrete made with recycled tire textile and steel fibers. MATEC Web of Conferences. 2013;6:07004.

3. Onuaguluchi O, Banthia N. Plant-based natural fibre reinforced cement composites: A review. Cem Conc Comp. 2016;68:96-108.

4. Messiry ME, Tarfawy SE, Deeb RE. Mechanical performance of cementitious composites reinforced with pultruded jute/polymeric matrix fabric. J Mat Sci \& Eng. 2015;4:173.

5. Ferreira SR, Lima PRL, Silva FA, et al. Effect of sisal fiber hornification on the fiber-matrix bonding characteristics and bending behavior of cement based composites. Key Eng Mat. 2014;600:421-432.

6. Barra B, Paulo B, Alves Junior C, et al. Effects of methane cold plasma in sisal fibers. Key Eng Mat. 2012;517:458-468.

7. Beraldo AL, Payá J, Monzó JM. Evaluation of compatibility between sugarcane straw particles and portland cement. Key Eng Mat. 2012;600:250-255
8. Hospodarova V, Singovszka E, Števulová N. Characterization of cellulosic fibres properties for their using in composites. Sol Sta Phen. 2016;244:146-152.

9. Nascimento Filho WB, Franco CR. Avaliação do potencial dos resíduos produzidos através do processamento agroindustrial no Brasil. Rev Virtual Quím. 2015;7(6):1969-1987.

10. Souza Junior FG, Pinto JC, Garcia FA, et al. Modification of coconut fibers with polyaniline for manufacture of pressure-sensitive devices. Pol Eng Sci. 2014;54(12):2887-2895.

11. Varela A, Oliveira G, Souza Jr FG, et al. New petroleum absorbers based on cardanol-furfuraldehyde magnetic nanocomposites. Pol Eng Sci. 2013;53(1):44-51.

12. Ahujaa D, Kaushika A, Chauhanb GS. Fractionation and physicochemical characterization of lignin from waste jute bags: Effect of process parameters on yield and thermal degradation. Int Jour Biol Macromol. 2017;97:403-410

13. Elias E, Costa R, Marques F, et al. Oil-spill cleanup: The influence of acetylated curaua fibers on the oil-removal capability of magnetic composites. Jour Ap Pol Sci. 2015;132(13):41732.

14. Silvertein RM, Webster FX. Indentificação Espectrometric de Compostos Orgânicos. 6th ed. Rio de Janeiro, Brasil: LTC; 2000.

15. Wei J, Meyer C. Utilization of rice husk ash in green natural fiber-reinforced cement composites: Mitigating degradation of sisal fiber. Cem Conc Res. 2016;81:94-111. 